\title{
The genesis of cerebellar GABAergic neurons: fate potential and specification mechanisms
}

\author{
Ketty Leto ${ }^{1,2}$, Chiara Rolando ${ }^{1,2}$ and Ferdinando Rossi ${ }^{1,2}$ \\ 1 Department of Neuroscience, Neuroscience Institute of Turin, University of Turin, Turin, Italy \\ ${ }^{2}$ Neuroscience Institute of the Cavalieri Ottolenghi Foundation, University of Turin, Turin, Italy
}

\section{Edited by:}

Salvador Martinez, University Miguel Hernandez, Spain

\section{Reviewed by:}

José A. Armengol, University Pablo de Olavide, Spain

Richard S. Nowakowski, Florida State University, USA

\section{*Correspondence:}

Ketty Leto, Neuroscience Institute of the Cavalieri Ottolenghi Foundation, University of Turin, Regione Gonzole 10, 10043 Orbassano, Turin, Italy. e-mail: ketty.leto@unito.it
All cerebellar neurons derive from progenitors that proliferate in two germinal neuroepithelia: the ventricular zone (VZ) generates GABAergic neurons, whereas the rhombic lip is the origin of glutamatergic types. Among VZ-derivatives, GABAergic projection neurons, and interneurons are generated according to distinct strategies. Projection neurons (Purkinje cells and nucleo-olivary neurons) are produced at the onset of cerebellar neurogenesis by discrete progenitor pools located in distinct VZ microdomains. These cells are specified within the VZ and acquire mature phenotypes according to cell-autonomous developmental programs. On the other hand, the different categories of inhibitory interneurons derive from a single population of Pax-2-positive precursors that delaminate into the prospective white matter (PWM), where they continue to divide up to postnatal development. Heterotopic/heterochronic transplantation experiments indicate that interneuron progenitors maintain full developmental potentialities up to the end of cerebellar development and acquire mature phenotypes under the influence of environmental cues present in the PWM. Furthermore, the final fate choice occurs in postmitotic cells, rather than dividing progenitors. Extracerebellar cells grafted to the prospective cerebellar white matter are not responsive to local neurogenic cues and fail to adopt clear cerebellar identities. Conversely, cerebellar cells grafted to extracerebellar regions retain typical phenotypes of cerebellar GABAergic interneurons, but acquire type-specific traits under the influence of local cues. These findings indicate that interneuron progenitors are multipotent and sensitive to spatiotemporally patterned environmental signals that regulate the genesis of different categories of interneurons, in precise quantities and at defined times and places.

Keywords: GABAergic interneuron, Pax-2, fate specification, heterotopic-heterochronic transplantation, Purkinje cell, ventricular zone, prospective white matter, Ascl-1

\section{INTRODUCTION}

The great attention paid in the last decades to the study of the basic mechanisms of neuropsychiatric disorders, such as autism, schizophrenia, epilepsy, and mental retardation, has contributed to highlight the pathogenic role played, together with other concurrent factors, by the unbalanced ratio between excitatory and inhibitory transmission in different CNS regions (Levitt et al., 2004; Di Cristo, 2007; Sun, 2007). GABAergic elements are most important to ensure an integrative and synchronized support for information processing in the brain (Möhler et al., 2004; Bartos et al., 2007). GABAergic signaling also regulates essential ontogenetic phases, from neuronal proliferation, migration, and differentiation (Owens and Kriegstein, 2002), to experience-dependent refinement of local circuits (Hensch, 2005). Inhibitory interneurons play a fundamental regulatory role on cortical development and plasticity, as they contribute to the onset, closure, and outcome of critical periods (Hensch, 2004). Therefore, understanding the developmental processes that regulate the generation of inhibitory interneurons and their assembly into neural networks is crucial to elucidate a broad range of physiologic and pathologic conditions.

Despite the wide variety of inhibitory interneurons present in the different subdivisions of the CNS, one major strategy applies to ensure the production of appropriate quantities of distinct interneuron subtypes. In most CNS regions, different categories of interneurons are generated by discrete pools of fate-restricted progenitors that become specified within defined germinal sites at precise developmental stages (Caspary and Anderson, 2003; Helms and Johnson, 2003; Bovetti et al., 2007; Batista-Brito et al., 2008; Wonders et al., 2008; Batista-Brito and Fishell, 2009). Recent findings, however, indicate that the variety of cerebellar GABAergic interneurons originates from a single population of multipotent progenitors that persist throughout embryonic and postnatal development and adopt different mature identities in response to local instructive cues. In the next sections we will first describe the general features of cerebellar neurogenesis and, then, we will focus on the mechanisms governing the generation of GABAergic projection neurons and interneurons.

\section{ORIGIN OF CEREBELLAR PHENOTYPES}

In the mouse, cerebellar histogenesis starts at embryonic day 9 (E9), after the establishment of regional specification and the formation of two germinal neuroepithelia that line the opening of the fourth ventricle: the rhombic lip (RL), located at the roof plate, and the ventricular zone (VZ), located at the inner germinal 
layer (Hatten and Heintz, 1995). The two neuroepithelia disappear at birth, but dividing progenitors emigrate from them and give rise to secondary germinal sites, where neurogenesis continues up to postnatal development. Hence, RL progenitors move along the cerebellar surface to form the external granular layer (EGL), whereas those of the $\mathrm{VZ}$ delaminate into the prospective white matter (PWM), which surrounds the deep nuclei and extends into the axis of the nascent folia.

According to the classical descriptions of cerebellar neurogenesis, the VZ was the source of deep cerebellar nuclei (DCN) neurons, Purkinje cells (PCs), and Golgi cells, whereas the EGL was the origin of granule neurons and inhibitory interneurons of the molecular layer (ML; Ramón Y Cajal, 1911; Altman, 1972). Later, it was unequivocally demonstrated that EGL progenitors exclusively generate granule cells (Hallonet et al., 1990; Hallonet and Le Douarin, 1993; Gao and Hatten, 1994). Furthermore, experiments using retroviral vectors (Zhang and Goldman, 1996a,b) together with clonal analyses (Mathis et al., 1997; Milosevic and Goldman, 2002, 2004; Mathis and Nicolas, 2003) showed that inhibitory interneurons derive from progenitors that proliferate in the PWM during late embryonic life and postnatal development. Finally, recent observations disclosed a precise neurochemical compartmentalization of cerebellar progenitors in the two primary germinal sites. RL precursors, which express the mouse homolog of Drosophila Atonal, Math-1, generate all glutamatergic types, including DCN projection neurons, unipolar brush cells, and granule cells, whereas $\mathrm{VZ}$ progenitors, distinguished by the expression of the pancreas transcription factor 1-a (Ptf1-a) produce all the GABAergic phenotypes, including PCs, nucleo-olivary projection neurons, and all the inhibitory interneurons (Hoshino et al., 2005; Machold and Fishell, 2005; Wang et al., 2005; Englund et al., 2006; Fink et al., 2006).

\section{NEUROGENESIS IN THE RL}

Two different regions can be distinguished in the RL: a rostral part that specifically gives rise to cerebellar glutamatergic neurons and a caudal one that generates the projection neurons of the major precerebellar nuclei (Alcántara et al., 2000; Wingate, 2001; Wang et al., 2005). A series of genetic fate mapping studies have finely described the temporal sequence of appearance of Math-1positive progenitors fated to different cerebellar districts (Wingate and Hatten, 1999; Lin et al., 2001; Wingate, 2001; Machold and Fishell, 2005; Machold et al., 2007). Math-1 expression begins in the murine RL at E9.5 (Akazawa et al., 1995) and is dynamically regulated by the antagonistic interaction between Notch1 signaling in the cerebellar primordium and the bone morphogenetic proteins secreted by the roof plate (Machold et al., 2007). Math-1 provides fundamental instructive information for the generation of cerebellar RL precursors. Mice carrying a Math-1 targeted deletion lack the rostral RL derivatives, whereas neurogenesis of caudal precursors is unaffected (Akazawa et al., 1995; Ben-Arie et al., 1997; Fünfschilling and Reichardt, 2002). Three main waves of fate-restricted cerebellar precursors sequentially leave the RL. The first one, from E10.5 to E12.5, gives rise to glutamatergic projection DCN neurons that migrate rostrally toward a subpial position, in the so called "nuclear transitory zone" (Machold and Fishell, 2005; Wang et al., 2005; Fink et al., 2006; Machold et al.,
2007). Secondly, progenitors of unipolar brush cells, glutamatergic interneurons of the granular layer $(\mathrm{GL})$, leave the germinal site between E14 and E21 (Nunzi et al., 2001, 2002; Sekerkovà et al., 2004) through two distinct streams of dorsally and rostrally migrating cells (Englund et al., 2006). Finally, a third migratory wave from the RL is composed by granule cell progenitors, which spread tangentially along the cerebellar surface to form the EGL in late embryogenesis and early postnatal life (Machold and Fishell, 2005). It has been recently shown that some granule cells may also derive from GFAP-expressing progenitors, which reside in the EGL during the first two postnatal weeks (Silbereis et al., 2010).

\section{NEUROGENESIS IN THE VZ}

Ventricular zone cells give origin to all cerebellar GABAergic neurons according to a two-step sequence: projection neurons (nucleo-olivary neurons and PCs) are generated at the onset of cerebellar neurogenesis by precursor cells that become specified within the germinal layer. Interneurons derive from $\mathrm{VZ}$ progenitors that delaminate into the PWM, where they continue to divide and take their final phenotypic choices (Figure 1; Zhang and Goldman, 1996a,b; Maricich and Herrup, 1999). Accordingly, the VZ comprises several cell subpopulations, distinguished by specific expression profiles of transcription factors and proneural genes that induce GABAergic specification or direct fate choices toward projection neurons or interneurons.

All VZ progenitors express the transcription factor Ptf1-a, which is required for the initial specification of the GABAergic

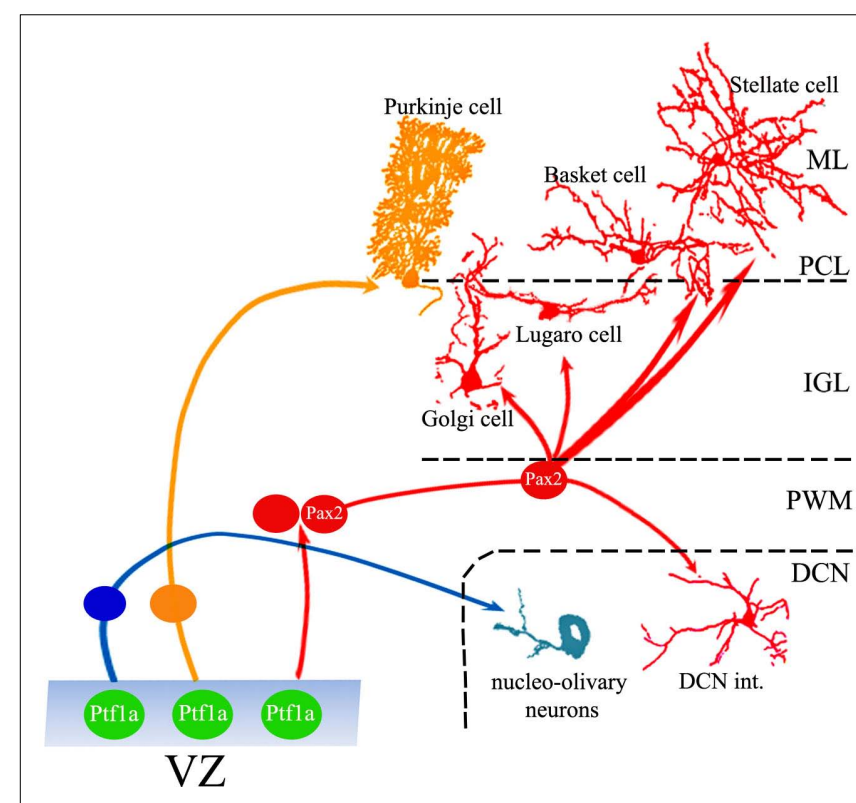

FIGURE 1 | Generation of cerebellar GABAergic neurons. Ptf1-a-positive progenitors in the VZ generate the full repertoire of GABAergic projection neurons and interneurons through different neurogenic strategies. While projection neurons are generated from precursors that proliferate and become specified within the $V Z$, interneurons derive from a population of dividing cells that originate in the $V Z$, but continue their neurogenic activity in the PWM, where they acquire mature identities under the influence of local cues. $\mathrm{ML}$, molecular layer; $\mathrm{PCL}$, Purkinje cell layer; IGL, internal granular layer; PWM, prospective white matter; DCN, deep cerebellar nuclei. 
lineage (Hoshino et al., 2005; Hoshino, 2006). In Ptf1-a knockout mice, some VZ progenitors migrate to the EGL and acquire granule cell phenotypes, indicating that Ptf1-a is needed to prevent VZ progenitors from engaging into a default granule cell developmental program (Pascual et al., 2007). The recent observation that Ptf1-a is mainly expressed in postmitotic VZ cells (Huang et al., 2010) suggests that this factor may act by inducing progenitors to leave the cycle and engage into GABAergic differentiation. This mechanism, however, cannot be immediately reconciled with the genesis of interneurons, which also derive from Ptf1-a-positive VZ cells that continue to proliferate in the PWM, where Ptf1-a is down-regulated.

While it is clear that Ptf1-a expression is directly involved in the choice between GABAergic or glutamatergic fates, the lineage relationships linking different VZ-derivatives are only partially known. For instance, although fate mapping analyses suggest that PCs, DCN neurons, and interneurons may be clonally related (Mathis et al., 1997), there is still no direct evidence that all GABAergic types actually derive from a single pool of VZ progenitors. It is now firmly established that the VZ comprises a mosaic of spatially discrete microdomains, distinguished by specific gene expression profiles, which are thought to be the origin of different populations or subpopulations of GABAergic neurons (Chizhikov et al., 2006; Morales and Hatten, 2006; Salsano et al., 2007; Sillitoe and Joyner, 2007; Zordan et al., 2008; Lundell et al., 2009; Mizuhara et al., 2009; Dalgard et al., 2011; Sudarov et al., 2011). Such VZ subdivisions are evident from the outset of cerebellar neurogenesis and persist throughout embryonic life, but undergo a characteristic evolution of their spatial arrangement (e.g., Zordan et al., 2008). Therefore, while the presence of microdomains is a landmark of VZ organization, it is still unclear whether VZ cells are a mosaic of distinct subpopulations that maintain steady properties throughout embryonic life, or represent a single pool of functionally dynamic cells, which modify their transcription profiles and acquire novel developmental potentialities at subsequent ontogenetic stages.

Ventricular zone cells are characterized by the expression of Neurogenin-1 (Ngn-1), Neurogenin-2 (Ngn-2), and Ascl-1 (Zordan et al., 2008). Ngn-1 and Ngn-2 are transiently expressed between E10.5 and E13.5 in distinct microdomains. In contrast, Ascl-1 is expressed during a longer time-window throughout the entire germinal layer (Zordan et al., 2008). Genetic fate mapping (Lundell et al., 2009; Kim et al., 2011) and examination of knockout animals (Dalgard et al., 2011) indicate that Ngn-1-positive progenitors are primarily fated to become projection neurons (notably PCs from E10.5 to E12.5), but they also contribute to generate interneurons at later times. Similarly, Ngn-2, which is a direct downstream target of the transcription factor complex Ptf1J in the spinal cord (Henke et al., 2009), also appears to be mainly involved in the generation of projection neurons (Florio et al., 2011). Finally, genetic fate mapping analysis with Ascl-1CreER ${ }^{T 2}$ transgenic mice indicates that this gene is transiently expressed by all VZ-derived neuronal and glial phenotypes, with different roles in their generation (Sudarov et al., 2011). Expression of Ascl-1 also occurs in PWM cells, including those derived from the VZ (Kim et al., 2008; Grimaldi et al., 2009; Sudarov et al., 2011), indicating that this factor also participates to neurogenic phenomena that occur at later ontogenetic stages (see below).

Among the VZ microdomains, some are related to the generation of different classes of PCs, whereas others define germinal regions destined to produce interneurons (Zordan et al., 2008; Lundell et al., 2009). To date, the only selective and specific marker for all maturing cerebellar GABAergic interneurons is Pax-2 (Maricich and Herrup, 1999). The first Pax-2-positive cells appear at E12.5 in a restricted region at the medial aspect of the VZ (Maricich and Herrup, 1999; Zordan et al., 2008). At this stage, the Pax-2-positive spatial domain is mutually exclusive with that of Ngn-1 and Ngn-2, while it is covered by Ascl-1 (Zordan et al., 2008). VZ progenitors also bear the cell surface marker Neph3, a downstream target of Ptf1-a (Mizuhara et al., 2009). Two subsets of Neph3-positive cells can be distinguished on the basis of high or low E-cadherin expression levels, which correspond to the progenitors of PCs and GABAergic interneurons, respectively (Mizuhara et al., 2009). Finally, another feature that can be used to discriminate between the two lineages is the differential sensitivity of VZ progenitors to sonic hedgehog (Shh): Shh signaling stimulates the proliferation of Blbp-positive radial glia (Anthony et al., 2005) to expand the pool of GABAergic interneuron progenitors, whereas it does not affect the generation of PCs (Huang et al., 2010).

\section{NEUROGENESIS AND GLIOGENESIS IN THE PWM}

The cerebellar PWM is a secondary germinal site, active during late embryonic life and postnatal development. The cellular composition of the PWM is complex, including cells with stem-like properties (Klein et al., 2005; Lee et al., 2005), dividing progenitors, and distinct categories of postmitotic elements at different maturation stages (Figure 2). Proliferating cells comprise progenitors that delaminated from the $\mathrm{VZ}$ or immigrated from extracerebellar sources. On the whole, PWM cells give rise to GABAergic interneurons, astrocytes, and oligodendrocytes (Figure 2; Zhang and Goldman, 1996b; Milosevic and Goldman, 2002, 2004; Grimaldi et al., 2009). Also in this case, however, the relationships linking these lineages are largely unclear.

In the PWM, Ascl-1 is active in two distinct cell populations: Olig2-positive cells show strong expression of Ascl-1, whereas Pax2-positive cells show weaker expression (Grimaldi et al., 2009). Consistent with the idea that this gene is involved in the genesis of interneurons and oligodendrocytes, both Ascl-1 null mice (Grimaldi et al., 2009) and conditional knockout mice (Sudarov et al., 2011) show a dramatic decrease of these cell types accompanied by increased numbers of astrocytes. Transplantation experiments (Grimaldi et al., 2009), corroborated by recent analysis of chick-quail chimeras (Mecklenburg et al., 2011), show that the majority of cerebellar oligodendrocytes actually derive from extracerebellar sources. Therefore, the loss of interneurons and the concomitant gain of astrocytes seen in Ascl-1 mutants suggest that these two lineages may be related, and Ascl-1 is involved in regulating their genesis. Overexpression of Ascl-1 at E15 yields high numbers of Pax-2-positive cells at the expense of astrocytes, suggesting that these two cell types derive from a common precursor whose fate choice toward the interneuron phenotype is directed by Ascl-1 (Grimaldi et al., 2009). In line with this view, fate mapping analysis of GFAPCreER ${ }^{\mathrm{T} 2}$ mice shows that cerebellar GABAergic 


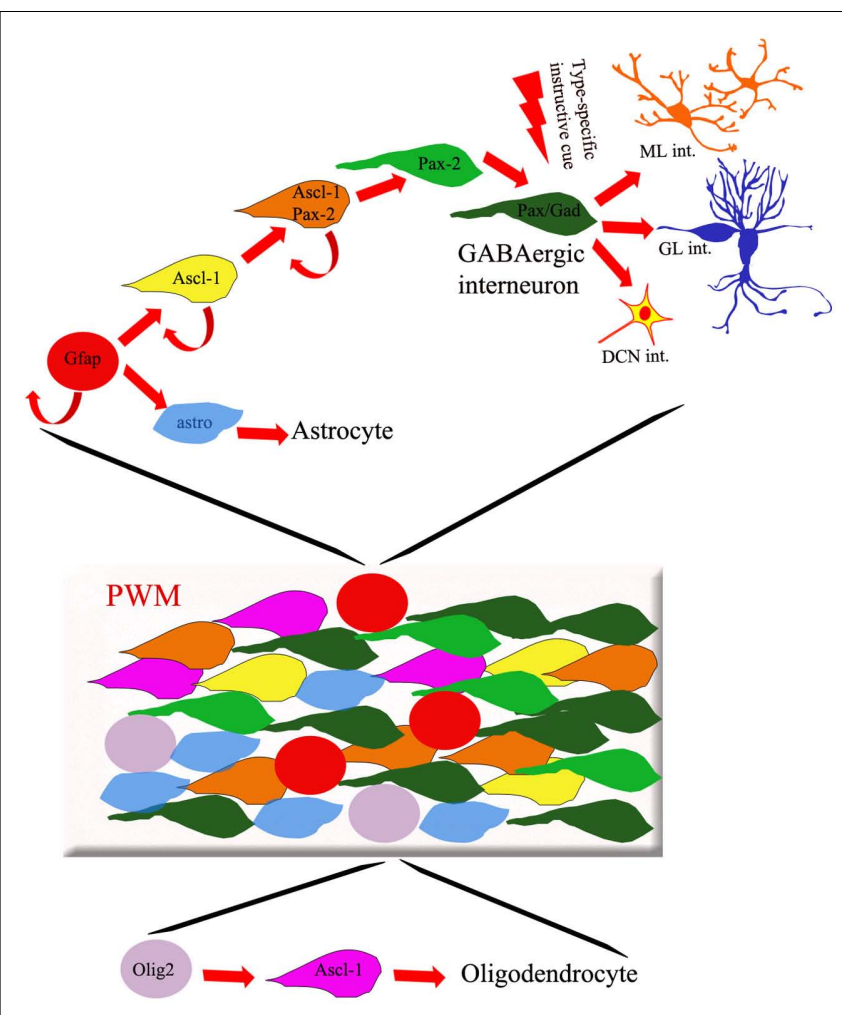

FIGURE 2 | Cellular composition of the PWM. The PWM contains different types of dividing progenitors and postmitotic cells fated to originate GABAergic interneurons, astrocytes, and oligodendrocytes. Whereas the latter cells derive from Olig-2-expressing progenitors (purple circles), GFAP-positive precursors (red circles) are thought to generate both inhibitory interneurons and astrocytes. The production of different subtypes of GABAergic interneurons is regulated by spatio-temporally patterned environmental influences, present in the PWM environment and acting on postmitotic Pax-2/Gad67-positive cells.

interneurons derive from a GFAP-positive precursor that could be shared with astrocytes (Figure 2, Silbereis et al., 2009). In spite of these observations, however, the unequivocal identification of a common progenitor for interneurons and astrocytes is still missing.

Among PWM cells, Pax-2 is the distinctive marker of the interneuron lineage. The original report indicated that Pax-2 is expressed by actively dividing interneuron progenitors (Maricich and Herrup, 1999). More recently, however, it has been shown that Pax-2 is upregulated when PWM progenitors enter their last cell division (Weisheit et al., 2006; Leto et al., 2009), implying that the generation of appropriate numbers of interneurons must be sustained by the proliferation of Pax-2-negative elements (Weisheit et al., 2006; Schilling et al., 2008). A recent study suggests that RBP$\mathrm{J}$ signaling converts a population of Sox-2-positive/Pax-2-negative precursors into Pax-2-positive interneuron progenitors (Komine et al., 2011). Other environmental factors, such as basic fibroblast growth factor (Lee et al., 2005), Shh (Huang et al., 2010), or thyroid hormones (Manzano et al., 2007), also contribute to regulate the production rates of interneurons. Similarly, cell-intrinsic mechanisms that control cell cycle progression and length also appear to be relevant for the proper outcome of these processes (Lee et al., 2009; Leto et al., 2011).

Following their last mitosis, Pax-2-positive cells remain in the PWM for a rather long time (up to several days), before moving to their final location in the DCN or cortex (Leto et al., 2009). During this period, the young interneurons progress in their maturation. For instance, expression of the GABA synthesizing enzyme glutamic acid decarboxylase only occurs about $24 \mathrm{~h}$ after the completion of the last cycle. The reason for such a long "waiting period" of young interneurons in the PWM is not clear. It must be noted that if Pax-2-expressing cells are positively committed to the GABAergic fate, young postmitotic interneurons are still able to switch their fate and adopt host-specific interneuron identities following heterotopic/heterochronic transplantation (see below; Leto et al., 2009). These considerations suggest that while Pax2 upregulation during the last mitosis marks the commitment of the cell toward the GABAergic interneuron destiny, the final fate choice toward distinct mature phenotypes is taken during the ensuing period, when the young interneurons sojourn in the PWM (Figure 2).

\section{SPATIO-TEMPORAL EVOLUTION OF THE DEVELOPMENTAL POTENTIAL OF CEREBELLAR PROGENITORS}

One of the most convenient ways to elucidate the relative contribution of cell intrinsic and environmental factors in the specification of neuronal phenotypes is to examine the fate of cells exposed to heterotopic and/or heterochronic environmental conditions. This approach is particularly suitable in the case of the genesis of cerebellar GABAergic neurons, given the peculiar time-sequence of phenotypic generation and the existence of two distinct germinal sites, the VZ and the PWM.

In this context, a first major question is whether postnatal progenitors, normally destined to generate interneurons, can switch their fates and become projection neurons when exposed to the environment of the embryonic cerebellar primordium. In a first study devoted to address this issue, morsels of postnatal (P4) and embryonic (E12) mouse cerebella were mixed together and co-grafted to the cerebellum or to the forth ventricle of adult hosts (Jankovski et al., 1996). To identify donor cells derived from either sources, postnatal tissues were isolated from transgenic mice expressing the LacZ reporter gene in different neuron populations. In such mixed grafts, the embryonic donor tissue developed typical minicerebellar structures (Sotelo and Alvarado-Mallart, 1991; Rossi et al., 1992), while postnatal cells were incorporated as individual elements within this framework. The latter cells exclusively differentiated into ML interneurons and granule cells (the P4 tissue also contained the EGL), indicating that they were strictly committed toward late-generated identities and not responsive to neurogenic cues provided by the embryonic milieu (Jankovski et al., 1996).

In a later study, to gain further insights on the spatio-temporal evolution of the fate potentiality of cerebellar progenitors, E12 or P4 donors tagged by green fluorescent protein (GFP) expression were dissociated to single cell suspensions and injected into the cerebral ventricles of E15 rat embryos or into E12 organotypic explants (Carletti et al., 2002). Donor cells of both ages engrafted in the cerebellum and in many extracerebellar sites of 
the recipient brain. Even in ectopic locations, however, these cells exclusively differentiated in cerebellar phenotypes, showing that cerebellar cells are strictly regionally specified already at E12. The phenotypic repertoires yielded by grafted cells differed according to their age: E12 donors developed the full repertoire of cerebellar phenotypes, including both projection neurons and interneurons, whereas postnatal cells only produced granule cells and GABAergic interneurons.

These experiments show that postnatal cerebellar progenitors are unable to adopt the identities of projection neurons, even when exposed to the embryonic cerebellar milieu. This suggests that the sequence of phenotypic generation during cerebellar neurogenesis is achieved by progressively reducing the developmental potentialities of progenitors. This outcome could be obtained either by restricting the differentiation properties of a single population of initially multipotent precursors, or by creating $a b$ initio distinct subsets of fate-restricted progenitors destined to generate different mature types at subsequent developmental stages. The early compartmentalization of the germinal neuroepithelia as well as direct experimental evidence indicates that the latter case applies to certain types of cerebellar neurons, and notably RL derivatives (Gao and Hatten, 1994; Alder et al., 1996). On the other hand, the clonal relationship that may link PCs and inhibitory interneurons (Mathis et al., 1997; Mathis and Nicolas, 2003) suggests that VZ progenitors are initially competent to generate the whole phenotypic repertoire, but their potential becomes restricted at more advanced developmental stages, when VZ cells emigrate to the PWM.

\section{ONE FOR ALL: MULTIPLE TYPES OF GABAergic INTERNEURONS FROM A SINGLE POOL OF PROGENITORS}

Inhibitory interneurons of the cerebellar cortex and DCN can be subdivided in several classes on the basis of their morphological, neurochemical, and functional properties (Schilling et al., 2008; Schilling, 2011). While basket and stellate cells in the ML are thought to be extreme variants of the same category (Rakic, 1972; Sultan and Bower, 1998; Koscheck et al., 2003), several subtypes of Golgi neurons populate the GL together with Lugaro, globular, and candelabrum cells (Geurtz et al., 2001; Lainé and Axelrad, 2002; Simat et al., 2007). DCN interneurons may also comprise different subtypes (Uussisari and Knöpfel, 2011), but these are still incompletely characterized (Schilling, 2011).

The entire repertoire of inhibitory interneurons derive from Pax-2-expressing cells that are continuously generated between E12.5 and P15, with a peak around P5, leading to the production of $75 \%$ of all the interneurons prior to $\mathrm{P} 7$ in the mouse (Weisheit et al., 2006; Leto et al., 2008). In the rodent cerebella the genesis of GABAergic interneurons is completed by the end of the second postnatal week, but in the rabbit Pax-2-positive cells continue to be generated for a considerably longer time by progenitors lying in the subpial layer (Ponti et al., 2008, 2010).

In all instances, GABAergic interneurons are produced during a considerably long period from a population of Pax-2-expressing cells. Different mechanisms could be envisaged to explain the unfolding of this process: (1) Pax-2-positive cells might comprise distinct subsets of fate-restricted progenitors committed to different interneuron phenotypes; (2) a single set of initially multipotent progenitors could be progressively restricted in their potentialities, so to generate distinct classes of interneurons in sequence; (3) a single population of multipotent progenitors may retain full potentialities throughout development and make phenotypic choices in response to extrinsic instructive information.

As already mentioned, analyses of clonal composition during cerebellar development suggest that Golgi, basket, and stellate cells may descend from a common lineage (Mathis et al., 1997; Mathis and Nicolas, 2003). These findings, however, do not rule out the existence of spatially segregated pools of fate-restricted cells or the progressive loss of developmental potentialities. To elucidate whether progenitors located in different regions of the postnatal cerebellum (e.g., the central white matter or the axial core of cortical lobules) have distinct developmental potentialities, cells isolated from the periventricular or the subcortical PWM of P1 cerebella were transplanted either to E15 embryos in utero or P7 pups in vivo. Donor cells from both origins yielded the same phenotypic repertoires, whose composition consistently depended on the age of the recipient cerebellum: the entire variety of interneurons was generated in embryonic recipients, whereas only lateborn ML interneurons were produced in postnatal cerebella (Leto et al., 2006). These results show that all interneuron progenitors have the same potentialities regardless of their position. To ask whether the fate potential of these progenitors is progressively restricted as development advances, the types of interneurons produced by E14 or P7 donor progenitors were compared following heterochronic transplantation to recipient cerebella of different embryonic or postnatal ages. Also in these experiments, grafted cells, regardless of their donor age, produced the same phenotypic repertoires, typical of the recipient age (Leto et al., 2006). Together, these experiments indicate that all interneuron progenitors share similar developmental potentialities, which are not dependent on their spatial location or ontogenetic stage. In addition, the consistent acquisition of host-specific identities in both heterotopic and heterochronic conditions indicates that such progenitors are multipotent and make their fate choices under the influence of environmental cues.

\section{SPECIFICATION OF GABAergic INTERNEURONS IN THE PWM}

The different categories of interneurons are generated during largely overlapping time windows, according to a precise insideout sequence, starting with cells destined to the DCN and progressing to those of the GL and ML (Miale and Sidman, 1961; Altman and Bayer, 1997; Yamanaka et al., 2004; Weisheit et al., 2006; Schilling et al., 2008). A salient feature of this process is the strict correlation between interneuron birthdates and placements: in the cortical layers earliest-born interneurons are located deeper, whereas later-born elements settle in progressively more superficial positions (Leto et al., 2009).

Heterochronically transplanted cerebellar cells become mature interneurons that match the phenotype and position of their endogenous counterparts generated at the time of transplantation, suggesting that the donor cells entrain into the recipient neurogenic mechanism and become specified soon after graft (Leto et al., 2009). In line with this conclusion, at short term after transplantation donor cells are found in the PWM and acquire mature traits according to the same times and modes of local interneurons (Leto 
et al., 2009). This behavior is not followed by other types of cerebellar cells, such as Purkinje or granule neurons, that settle into the recipient tissue through unusual routes and differentiate according to cell-intrinsic mechanisms (Sotelo and Alvarado-Mallart, 1991; Grimaldi et al., 2005; Carletti et al., 2008; Williams et al., 2008).

Interestingly, also the temporal pattern of Ascl-1 expression in interneuron progenitors correlates with their final placement in the cerebellar cortex (Sudarov et al., 2011). Similar to Pax2, Ascl-1 is expressed by interneuron progenitors close to their final cycle (Sudarov et al., 2011). On the whole, these findings suggest that phenotypic specification and laminar fate of interneuron progenitors are determined at the time of their last mitosis. Quite surprisingly, however, this conclusion is contradicted by the behavior of juvenile interneurons grafted to heterochronic hosts (Leto et al., 2009). Postmitotic interneurons, identified by BrdU incorporation before cell dissection or by FACsorting of GAD67GFP-tagged cells (Yamanaka et al., 2004), are still able to switch their fate and adopt identities typical of the recipient age (Leto et al., 2009). As a consequence, although phenotype and position are temporally related to the cell birthdate, specification to distinct mature types actually occurs after the completion of the last cell division.

Even if the final fate choice occurs in postmitotic cells, mechanisms that operate during the last cycle may influence the developmental potential of interneuron precursors. For instance, inactivation of Xrcc1, a DNA repair protein, produces a dramatic loss of GABAergic interneurons due to p53-dependent cell cycle arrest occurring at the moment when progenitor cells start to differentiate (Lee et al., 2009). Likewise, mice lacking the G1-phase active protein cyclin D2 show a severe loss of stellate cells (Huard et al., 1999) and a severe delay in interneuron maturation (Leto et al., 2011). At least for the latter mice, however, the progenitors' capacity to acquire distinct mature phenotypes is not affected (Leto et al., 2011). Therefore, precise regulation of cell cycle dynamics appears to be more relevant in determining the rate of interneuron production rather than controlling phenotype specification.

The neurogenic properties of the PWM milieu have been investigated by transplantation of postnatal cerebellar cells to adult hosts, after the end of interneuron genesis. In these experiments, dissociated donor cells exclusively generated stellate neurons positioned in the outermost regions of the ML. On the contrary, solid pieces of PWM implanted in the recipient parenchyma yielded high quantities of interneurons scattered throughout the white matter and cortical layers. Most interestingly, these solid PWM grafts exposed to the non-neurogenic environment of the adult cerebellum, yielded interneuron repertoires consistent with the donor age: P1 tissue produced both GL and ML interneurons, whereas P7 tissue exclusively generated the latter types (Leto et al., 2009). Thus, in the absence of local neurogenic information in the adult host cerebellum (Grimaldi and Rossi, 2006), the donor PWM appears to be competent to generate different types interneurons in a stage-specific manner.

The nature of the instructive signals contained in the PWM milieu remains elusive. Gene expression analysis of interneuron progenitors failed to reveal distinct subtype-specific expression patterns, further confirming the view that the variety of GABAergic interneurons is mainly determined by local signaling
(Glassmann et al., 2009). The latter likely includes the concurrent contribution of cell-cell interactions, diffusible cues, and position-dependent information provided by components of the extracellular matrix (Leto et al., 2008, 2010; Schilling et al., 2008; Schilling, 2011). In any case, the molecular mediators of these processes and their mechanisms of action remain to be established.

\section{NEUROGENIC PROPERTIES OF THE PWM AND ADAPTIVE CAPABILITIES OF INTERNEURON PROGENITORS}

The findings discussed in the previous sections indicate that the generation of different types of interneurons depends on the sensitivity of the progenitors to instructive cues present in their surrounding microenvironment. Another suitable way to investigate the neurogenic properties of the developing cerebellar milieu is the transplantation of foreign progenitors. Following in utero injection, E12 neocortical cells engraft in many different CNS regions, including the cerebellum (Carletti et al., 2004). However, while these donors acquire site-specific identities in different telencephalic sites, in the cerebellum they fail to develop any cerebellar-specific traits and show poor integration capabilities.

More recently, it was reported that progenitors from the forebrain subventricular zone transplanted to the postnatal PWM acquire anatomical and neurochemical features of cerebellar interneurons (Milosevic et al., 2008). Later on, however, comparison of the fates of progenitors isolated from different sites along the neuraxis (lateral ganglionic eminence, telencephalic subventricular zone, ventral mesencephalon, dorsal spinal cord) led to partially different conclusions (Rolando et al., 2010). In the latter transplants, extracerebellar progenitors did not express cerebellarspecific regulatory genes and failed to acquire clear cerebellar phenotypes and positions consistent with the recipient age. Interestingly, some of the grafted neurons developed peculiar morphological features, such as particular arrangements of their dendrites, as a result of their interaction with the surrounding architecture of host cerebellum. Hence, while extracerebellar cells are not sensitive to neurogenic signals provided by the PWM, they are able to adapt to local constraints and acquire some mature traits that are typical of the recipient tissue.

Heterotopic transplantation to extracerebellar sites is also a useful tool to investigate the intrinsic developmental properties of interneuron progenitors. As remarked above, cerebellar cells implanted in utero settle in wide areas of the recipient CNS, but exclusively acquire cerebellar phenotypes (Carletti et al., 2002). Namely, both embryonic and postnatal donors produce different types of GABAergic interneurons that can be readily assigned to distinct categories by their morphologies and neurochemical profiles. The variety of interneurons produced in foreign engraftment sites suggests that, although cerebellar progenitors are strictly committed to regional fates, even in ectopic locations their acquisition of different mature identities may still be influenced by environmental cues.

To address this question, GFP-tagged cells from the cerebella of $\mathrm{P} 1$ and $\mathrm{P} 7$ rats were injected in the ventricles of E15 rat embryos in utero, and their fate was examined 1 month posttransplantation. $\mathrm{P} 1$ donor cells engrafted in different places along the neuraxis, including neocortex, striatum, hippocampus, thalamus, hypothalamus, midbrain, and brainstem. Conversely, P7 
cells were never found in the telencephalon, but they were mainly distributed in the midbrain and brainstem (Figure 3A). In line with previous findings, donor cells of both ages differentiated in glia, granule cells, and inhibitory interneurons, with larger fractions of neurons in the most caudal engraftment sites (Jankovski et al., 1996; Carletti et al., 2002). The phenotype of transplanted GABAergic interneurons was also evaluated by assessing their expression of markers specific for different categories: NeuN and calretinin (CR) for DCN interneurons, Pax-2 or neurogranin for Golgi neurons (Figure 3B), parvalbumin (PV) for ML interneurons (Figure 3C, Bastianelli, 2003; Singec et al., 2003; Leto et al., 2008; Rolando et al., 2010). Both P1 and P7 donors developed typical interneuron morphologies and expressed various markers in different host regions, confirming that they could acquire structural and neurochemical features distinctive of different categories of cerebellar interneurons even in ectopic positions. Among such donor-derived interneurons, 66.8\% expressed PV (121/181 cells; $n=10$ animals), $28.3 \%$ was CR-positive (49/173 cells; $n=10$ animals), and $21.3 \%$ was NeuN-positive $(37 / 174 ; n=10$ animals). Importantly, no grafted cells ever co-expressed PV and $\mathrm{CR}$, confirming that these markers represent mutually exclusive phenotypic traits even in ectopically placed cells.

To understand whether the phenotypes acquired by heterotopically grafted interneurons were influenced by local cues, the neurochemical profiles of individual donor interneurons were compared to those of nearby host neurons. The vast majority PVpositive donor interneurons (112/121 cells) integrated in recipient regions where no endogenous PV-positive cells were present (Figures 3D,G). On the contrary, most of CR-positive $(83.7 \%$, out of 43/49 cells; Figures 3E,G) and NeuN-positive interneurons (96.5\%, 109/113 cells; Figures 3F,G) were consistently intermingled with host cells expressing the same marker. These observations indicate that although cerebellar interneuron progenitors develop cerebellar-specific phenotypes in a cell-autonomous manner regardless of their ectopic location, at least some of their phenotypic traits may be influenced by local cues. This appears particularly evident for markers typical of DCN or GL interneurons, such as NeuN or CR, but not for PV, which is distinctive of ML interneurons. This suggests that the acquisition of DCN or GL interneuron identities is directed by external instructive information, whereas the ML phenotype can be acquired by executing some sort of default cell-intrinsic program.

\section{CONCLUDING REMARKS}

The findings reported in the previous sections indicate that distinct strategies sustain the generation of cerebellar GABAergic projection neurons and interneurons (Figure 1). All cerebellar GABAergic phenotypes derive from a single germinal layer, the VZ. Similar to other CNS sites, projection neurons are generated first, at the onset of neurogenesis, from progenitors that proliferate within the neuroepithelium. Their specification and differentiation are regulated by transcriptional programs expressed in specific subsets of VZ cells, distributed in discrete microdomains. As a consequence, the intrinsic fate potential of such cells is restricted at early ontogenetic stages and they acquire mature phenotypes essentially by unfolding cell-autonomous programs.

GABAergic interneurons derive from a single population of dividing cells that originate in the VZ, but continue their neurogenic activity in a secondary germinal site, the PWM. Transplantation experiments show that interneuron precursors are not restricted in their fate potential, but maintain the ability for generating the complete phenotypic repertoire up to the latest developmental stages. In addition, early postmitotic cells grafted to heterotopic/heterochronic recipients are still able to switch their fate and adopt host-specific interneuron identities. The latter feature suggests that upregulation of Pax-2, during the last cell
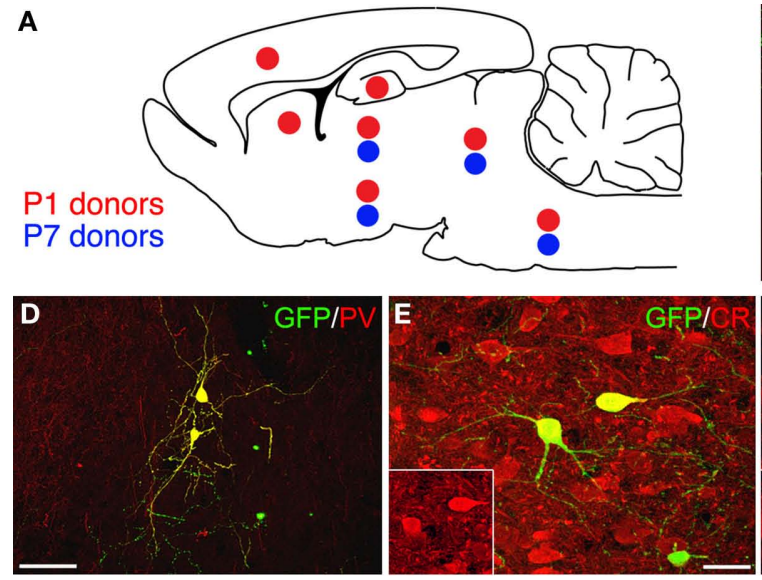

FIGURE 3 | Distribution and neurochemical profile of cerebellar progenitors heterotopically grafted to embryonic hosts in utero. (A) Distribution of P1 (red dots) and P7 (blue dots) GFP-expressing donor cells: P1 cells integrate widely along the neuroaxis, whereas P7 donors are found only in midbrain and brainstem. (B,C) Examples of GFP-positive cerebellar progenitors expressing Pax-2 and PV (red). (D-F) The ectopically positioned cells (green) express markers of cerebellar interneurons, such as PV [(D), red],
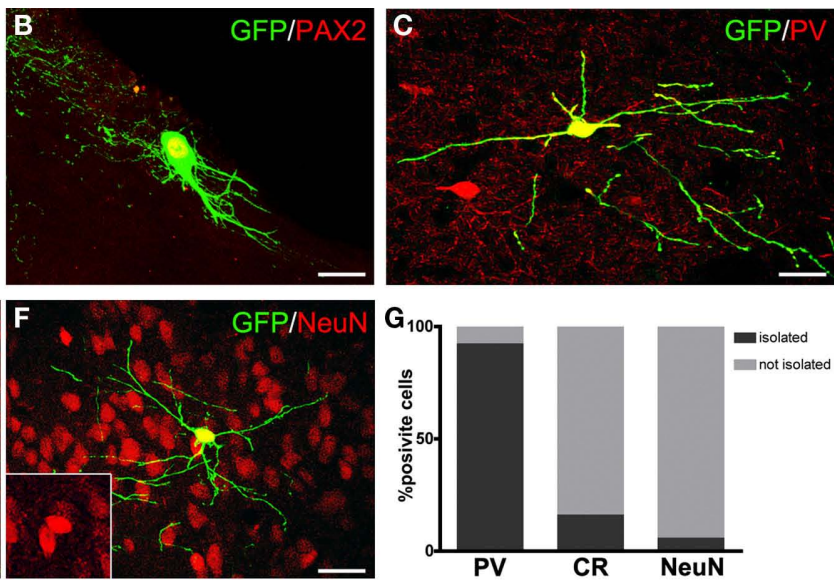

CR [(E), red], and NeuN [(F), red]. (G) Quantitative analysis of the distribution of transplanted interneurons expressing $\mathrm{PV}, \mathrm{CR}$, or NeuN in relation to the expression of the same markers in neighboring host neurons: the majority of PV-positive cells engraft in host regions where PV is not expressed (D,G), whereas CR- (E,G) or NeuN-positive interneurons $(\mathbf{F}, \mathbf{G})$ are most often intermingled with host neurons expressing the same marker. Scale bars: in $(\mathbf{B}, \mathbf{C}, \mathbf{E}, \mathbf{F})=20 \mu \mathrm{m} ;(\mathbf{D})=50 \mu \mathrm{m}$. 
cycle, marks the acquisition of the inhibitory interneuron identity, whereas the development of the distinctive traits of the different interneuron categories occurs in postmitotic interneurons under the influence of instructive environmental cues. The acquisition of site-specific traits by cerebellar interneurons that engrafted in extracerebellar sites is also consistent with this view.

Although this model of the genesis of cerebellar GABAergic neurons is corroborated by several lines of evidence, many fundamental questions remain unanswered. For instance, the lineage relationships linking projection neurons and interneurons are unclear. In this context, it is particularly important to define whether the same progenitors can generate both projection neurons and interneurons, or if these two categories derive from distinct pools of VZ cells. Another important issue refers to the nature and the source of the instructive cues present in the PWM milieu. We have recently proposed that specific signals may be issued by different cell populations that surround the PWM at subsequent ontogenetic stages (Leto et al., 2008, 2010). Although this idea is consistent with some preliminary observations, the mediators of the underlying signaling and their mechanisms of action remain obscure. Likewise, the composition and the neurogenic properties of the PWM itself have to be explored and characterized. Indeed, proliferating progenitors and young postmitotic interneurons pass through crucial decision points and

\section{REFERENCES}

Akazawa, C., Ishibashi, M., Shimizu, C., Nakanishi, S., and Kageyama, R. (1995). A mammalian helix-loophelix factor structurally related to the product of Drosophila proneural gene atonal is a positive transcriptional regulator expressed in the developing nervous system. J. Biol. Chem. 270, 8730-8738.

Alcántara, S., Ruiz, M., De Castro, F., Soriano, E., and Sotelo, C. (2000). Netrin 1 acts as an attractive or as a repulsive cue for distinct migrating neurons during the development of the cerebellar system. Development 127, 1359-1372.

Alder, J., Cho, N. K., and Hatten, M. E. (1996). Embryonic precursor cells from the rhombic lip are specified to a cerebellar granule neuron identity. Neuron 17, 389-399.

Altman, J. (1972). Postnatal development of the cerebellar cortex in the rat. I. The external germinal layer and the transitional molecular layer. J. Comp. Neurol. 145, 353-398.

Altman, J., and Bayer, S. A. (1997). Development of the Cerebellar System in Relation to its Evolution, Structures and Functions. Boca Raton, FL: CRC.

Anthony, T. E., Mason, H. A., Gridley, T., Fishell, G., and Heintz, N. (2005). Brain lipid-binding protein is a direct target of Notch signaling in radial glial cells. Genes Dev. 19, 1028-1033.
Bartos, M., Vida, I., and Jonas, P. (2007). Synaptic mechanisms of synchronized gamma oscillations in inhibitory interneuron networks. Nat. Rev. Neurosci. 8, 45-56.

Bastianelli, E. (2003). Distribution of calcium-binding proteins in the cerebellum. Cerebellum 2, 242-262.

Batista-Brito, R., Close, J., Machold, R., and Fishell, G. (2008). The distinct temporal origins of olfactory bulb interneuron subtypes. J. Neurosci. 28, 3966-3975.

Batista-Brito, R., and Fishell, G. (2009). The developmental integration of cortical interneurons into a functional network. Curr. Top. Dev. Biol. 87, 81-118.

Ben-Arie, N., Bellen, H. J., Armstrong, D. L., McCall, A. E., Gordadze, P. R., Guo, Q., Matzuk, M. M., and Zoghbi, H. Y. (1997). Math1 is essential for genesis of cerebellar granule neurons. Nature 390, 169-172.

Bovetti, S., Peretto, P., Fasolo, A., and De Marchis, S. (2007). Spatiotemporal specification of olfactory bulb interneurons. J. Mol. Histol. 38, 563-569.

Carletti, B., Grimaldi, P., Magrassi, L., and Rossi, F. (2002). Specification of cerebellar progenitors following heterotopic/heterochronic transplantation to the embryonic CNS in vivo and in vitro. J. Neurosci. 22, 7132-7146.

undergo profound developmental changes being immersed in this environment. However, most of the cellular/molecular interactions that govern these processes and determine their outcome are still totally unknown. Finally, the reason why cerebellar interneurons are generated through such a peculiar mechanism has to be explained. The recent discovery of the prominent role played by the outer subventricular zone in the evolution of mammalian neocortex (Lui et al., 2011) highlights a potential adaptive function of secondary germinal sites, such as the cerebellar PWM, in providing a powerful ontogenetic mechanism to expand certain neuron populations and match their production rates to the requirements of rapidly evolving circuitries. All these issues, however, still await sound demonstration and, in spite of the considerable knowledge that we acquired about the developmental mechanisms of cerebellar interneurons, a long way has still to be run before we can really understand the fine nature of these processes.

\section{ACKNOWLEDGMENTS}

This work was supported by grants from Ministero dell'Università e della Ricerca (PRIN 2009 prog. nr. 2009 TBCZJB), Compagnia di San Paolo (Neurotransplant Project 2008; GABAGEN project 2009), Regione Piemonte (Project A14/05; Ricerca Sanitaria Finalizzata, 2009), and Ataxia UK. Ketty Leto is supported by a fellowship from Compagnia di San Paolo.

Carletti, B., Grimaldi, P., Magrassi, L., and Rossi, F. (2004). Engraftment and differentiation of neocortical progenitor cells transplanted to the embryonic brain in utero. J. Neurocytol. 33, 309-319.

Carletti, B., Williams, I. M., Leto, K., Nakajima, K., Magrassi, L., and Rossi, F. (2008). Time constraints and positional cues in the developing cerebellum regulate Purkinje cell placement in the cortical architecture. Dev. Biol. 317, 147-160.

Caspary, T., and Anderson, K. V. (2003). Patterning cell types in the dorsal spinal cord: what the mouse mutants say. Nat. Rev. Neurosci. 4, 289-297.

Chizhikov, V. V., Lindgren, A. G., Currle, D. S., Rose, M. F., Monuki, E. S., and Millen, K. J. (2006). The roof plate regulates cerebellar celltype specification and proliferation. Development 133, 2793-2804.

Dalgard, C. L., Zhou, Q., Lundell, T. G., and Doughty, M. L. (2011). Altered gene expression in the emerging cerebellar primordium of Neurog1/- mice. Brain Res. 1388, 12-21.

Di Cristo, G. (2007). Development of cortical GABAergic circuits and its implications for neurodevelopmental disorders. Clin. Genet. 72, $1-8$.

Englund, C. M., Kowalczyk, T., Daza, R. A. M., Dagan, A., Lau, C., Rose, M. F., and Hevner, R. F. (2006). Unipolar brush cells of the cerebellum are produced in the rhombic lip and migrate through developing white matter. J. Neurosci. 26, 9184-9195.

Fink, A. J., Englund, C., Daza, R. A. M., Pham, D., Lau, C., Nivison, M., Kowalczk, T., and Hevner, R. F. (2006). Development of the deep cerebellar nuclei: transcription factors and cell migration from the rhombic lip. J. Neurosci. 26, 3066-3076.

Florio, M., Leto, K., Muzio, L., Tinterri, A., Badaloni, A., Croci, L., Zordan, P., Barili, V., Albieri, I., Guillemot, F., Rossi, F., and Consalez, G. G. (2011). Neurog2 regulates progenitor cell cycle progression and Purkinje cell dendritogenesis in cerebellar development. Mol. Mech. Neurosci. abstr. 56.

Fünfschilling, U., and Reichardt, L. F. (2002). Cre-mediated recombination in rhombic lip derivatives. Genesis 33, 160-169.

Gao, W. Q., and Hatten, M. E. (1994). Immortalizing oncogenes subvert the establishment of granule cell identity in developing cerebellum. Development 120, 1059-1070.

Geurtz, F. J., Timmermans, J., Shigemoto, R., and De Schutter, E (2001). Morphological and neurochemical differentiation of large granular layer interneurons in the adult rat cerebellum. Neuroscience 104, 499-512. 
Glassmann, A., Topka, S., WangEckardt, L., Anders, S., Weisheit, G., Endl, E., Zimmer, A., and Schillig, K. (2009). Basic molecular fingerprinting of immature cerebellar cortical inhibitory interneurons and their precursors. Neuroscience 159, 69-82.

Grimaldi, P., Carletti, B., Magrassi, L., and Rossi, F. (2005). Fate restriction and developmental potential of cerebellar progenitors. Transplantation studies in the developing CNS. Prog. Brain Res. 148, 57-68.

Grimaldi, P., Parras, C., Guillemot, F., Rossi, F., and Wassef, M. (2009). Origins and control of the differentiation of inhibitory interneurons and glia in the cerebellum. Dev. Biol. 328, 422-433.

Grimaldi, P., and Rossi, F. (2006). Lack of neurogenesis in the adult rat cerebellum after Purkinje cell degeneration and growth factor infusion. Eur. J. Neurosci. 23, 2656-2668.

Hallonet, M. E., and Le Douarin, N. M. (1993). Tracing neuroepithelial cells of the mesencephalic and metencephalic alar plates during cerebellar ontogeny in quail-chick chimaeras. Eur. J. Neurosci. 5, 1145-1155.

Hallonet, M. E., Teillet, M. A., and Le Douarin, N. M. (1990). A new approach to the development of the cerebellum provided by the quailchick marker system. Development 108, 19-31.

Hatten, M. E., and Heintz, N. (1995). Mechanisms of neural patterning and specification in the developing cerebellum. Annu. Rev. Neurosci. 18, 385-408.

Helms, A. W., and Johnson, J. E. (2003). Specification of dorsal spinal cord interneurons. Curr. Opin. Neurobiol. 13, 42-49.

Henke, R. M., Savage, T. K., Meredith, D. M., Glasgow, S. M., Hori, K., Dumas, J., MacDonald, R. J., and Johnson, J. E. (2009). Neurog2 is a direct downstream target of the Ptfla-Rbpj transcription complex in dorsal spinal cord. Development 136, 2945-2954.

Hensch, T. K. (2004). Critical period regulation. Annu. Rev. Neurosci. 27, 549-579.

Hensch, T. K. (2005). Critical period plasticity in local cortical circuits. Nat. Rev. Neurosci. 6, 877-888.

Hoshino, M. (2006). Molecular machinery governing GABAergic neuron specification in the cerebellum. Cerebellum 5, 193-198.

Hoshino, M., Nakamura, S., Mori, K., Kawauchi, T., Terao, M., Nishimura, Y. V., Fukuda, A., Fuse, T., Matsuo, N., Sone, M., Watanabe, M., Bito, H., Terashima, T., Wright, C. V. E., Kawaguchi, Y., Nakao, K., and
Nabeshima, Y. I. (2005). 'Ptfla, a bHLH transcriptional gene, defines GABAergic neuronal fates in cerebellum. Neuron 47, 201-213.

Huang, X., Liu, J., Ketova, T., Fleming, J. T., Grover, V. K., Cooper, M. K., Litingtung, Y., and Chiang, C. (2010). Transventricular delivery of Sonic hedgehog is essential to cerebellar ventricular zone development. Proc. Natl. Acad. Sci. U.S.A. 107, 8422-8427.

Huard, J. M., Forster, C. C., Carter, M. L., Sicinski, P., and Ross, M. E. (1999). Cerebellar histogenesis in mice lacking cyclin D2. Development 126, 1927-1935.

Jankovski, A., Rossi, F., and Sotelo, C. (1996). Neuronal precursors in the postnatal mouse cerebellum are fully committed cells: evidence from heterochronic transplantation. Eur. J. Neurosci. 8, 2308-2320.

Kim, E. J., Battiste, J., Nakagawa, Y., and Johnson, J. E. (2008). Ascll (Mash1) lineage cells contribute to discrete cell populations in CNS architecture. Mol. Cell. Neurosci. 38, 595-606.

Kim, E. J., Hori, K., Wyckoff, A., Dickel, L. K., Koundakjian, E. J., Goodrich, L. V., and Johnson, J. E. (2011). Spatiotemporal fate map of neurogenin1 (Neurog1) lineages in the mouse central nervous system. J. Comp. Neurol. 519, 1355-1370.

Klein, C., Butt, S. J. B., Machold, R. P., Johnson, J. E., and Fishell, G. (2005). Cerebellum and forebrain derived stem cells possess intrinsic regional character. Development 132, 4497-4508.

Komine, O., Nagaoka, M., Hiraoka, Y., Hoshino, M., Kawaguchi, Y., Pear, W. S., and Tanaka, K. (2011). RBPJ promotes the maturation of neuronal progenitors. Dev. Biol. 354, 44-54.

Koscheck, T., Weyer, A., Schilling, R. L., and Schilling, K. (2003). Morphological development and neurochemical differentiation of cerebellar inhibitory interneurons in microexplants cultures. Neuroscience 116, 973-984.

Lainé, J., and Axelrad, H. (2002). Extending the cerebellar Lugaro cell class. Neuroscience 115, 363-374.

Lee, A., Kessler, J. D., Read, T. A., Kaiser, C., Corbeil, D., Huttner, W. B., Johnson, J. E., and Wechsler-Reya, R. J. (2005). Isolation of neural stem cells from the postnatal cerebellum. Nat. Neurosci. 6, 723-729.

Lee, Y., Katyal, S., Li, Y., El-Khamisy, S. F., Russell, H. R., Caldecott, K. W., and McKinnon, P. J. (2009). Genesis of cerebellar interneurons and the prevention of neural DNA damage require XRCC1. Nat. Neurosci. 12, 973-980.

Leto, K., Bartolini, A., Di Gregorio, A. Imperiale, D., De Luca, A., Parmigiani, E., Filipkowski, R. K., Kaczmarek, L., and Rossi, F. (2011). Modulation of cell-cycle dynamics is required to regulate the numbers of cerebellar GABAergic interneurons and their rhythm of maturation. Development 138, 3463-3472.

Leto, K., Bartolini, A., and Rossi, F (2008). Development of cerebellar GABAergic interneurons: origin and shaping of the "minibrain" local connections. Cerebellum 7, 523-529.

Leto, K., Bartolini, A., and Rossi, F. (2010). The prospective white matter: an atipica neurogenic niche in the developing cerebellum. Arch. Ital. Biol. 148, 137-146.

Leto, K., Bartolini, A., Yanagawa, Y., Obata, K., Magrassi, L., Schilling, K., and Rossi, F. (2009). Laminar fate and phenotype specification of cerebellar GABAergic interneurons. J. Neurosci. 29, 7079-7091.

Leto, K., Carletti, B., Williams, I. M., Magrassi, L., and Rossi, F. (2006). Different types of cerebellar GABAergic interneurons originate from a common pool of multipotent progenitor cells. J. Neurosci. 26, 11682-11694.

Levitt, P., Eagleson, K. L., and Powell, E. M. (2004). Regulation of neocortical interneuron development and the implications for neurodevelopmental disorders. Trends Neurosci. 27, 400-406.

Lin, J. C., Cai, L., and Cepko, C. L. (2001). The external granule layer of the developing chick cerebellum generates granule cells and cells of the isthmus and rostral hindbrain. $J$. Neurosci. 21, 159-168.

Lui, J. H., Hansen, D. V., and Kriegstein, A. R. (2011). Development and evolution of the human neocortex. Cell 146, 18-36.

Lundell, T. G., Zhou, Q., and Doughty, M. L. (2009). Neurogenin1 expression in cell lineages of the cerebellar cortex in embryonic and postnatal mice. Dev. Dyn. 238, 3310-3325

Machold, R. P., and Fishell, G. (2005) Math1 is expressed in temporally discrete pools of cerebellar rhombiclip neural progenitors. Neuron 48, 17-24.

Machold, R. P., Kittell, D. J., and Fishell, G. J. (2007). Antagonism between Notch and bone morphogenetic protein receptor signaling regulates neurogenesis in the cerebellar rhombic lip. Neural Dev. 2, 5 .
Manzano, J., Cuadrado, M., Morte, B., and Bernal, J. (2007). Influence of thyroid hormone and thyroid hormone receptors in the generation of cerebellar gamma-aminobutyric acid-ergic interneurons from precursor cells. Endocrinology 148, 5746-5751.

Maricich, S. M., and Herrup, K. (1999). Pax-2 expression defines a subset of GABAergic interneurons and their precursors in the developing murine cerebellum. J. Neurobiol. 41, 281-294.

Mathis, L., Bonnerot, C., Puelles, L. and Nicolas, J. F. (1997). Retrospective clonal analysis of the cerebellum using genetic laacZ/lacZ mouse mosaics. Development 124, 4089-4104.

Mathis, L., and Nicolas, J. F. (2003). Progressive restriction of cell fates in relation to neuroepithelial cell mingling in the mouse cerebellum. Dev. Biol. 258, 20-31.

Mecklenburg, N., Garcia-López, R., Puelles, E., Sotelo, C., and Martinez, S. (2011). Cerebellar oligodendroglial cells have a mesencephalic origin. Glia. 59, 1946-1957.

Miale, I. R., and Sidman, R. L. (1961). An autoradiographic analysis of histogenesis in the mouse cerebellum. Exp. Neurol. 4, 277-296.

Milosevic, A., and Goldman, J. E. (2002). Progenitors in the postnatal cerebellar white matter are antigenically heterogeneous. J. Comp. Neurol. 452, 192-203.

Milosevic, A., and Goldman, J. E. (2004). Potential of progenitors from postnatal cerebellar neuroepithelium and white matter: lineage specified vs multipotent fate. Mol. Cell. Neurosci. 26, 342-353.

Milosevic, A., Noctor, S. C., MartinezCerdeno, V., Kriegstein, A. R. and Goldman, J. E. (2008) Progenitors from the postnatal forebrain subventricular zone differentiate into cerebellar-like interneurons and cerebellar-specific astrocytes upon transplantation. Mol. Cell. Neurosci. 39, 324-334.

Mizuhara, E., Minaki, Y., Nakatani, T., Kumai, M., Inoue, T., Muguruma, K. Sasai, Y., and Ono, Y. (2009). Purkinje cells originate from cerebellar ventricular zone progenitors positive for Neph3 and E-cadherin. Dev. Biol. 338, 202-214.

Möhler, H., Fritschy, J. M., Crestani, F., Hensch, T., and Rudolph, U. (2004). Specific GABA(A) circuits in brain development and therapy. Biochem. Pharmacol. 68, 1685-1690. 
Morales, D., and Hatten, M. E. (2006). Molecular markers of neuronal progenitors in the embryonic cerebellar anlage. J. Neurosci. 26, 12226-12236.

Nunzi, M. G., Birnstiel, S., Bhattacharyya, B. J., Slater, N. T., and Mugnaini, E. (2001). Unipolar brush cells form a glutamatergic projection system within the mouse cerebellar cortex. J. Comp. Neurol. 434, 329-341.

Nunzi, M. G., Shigemoto, R., and Mugnaini, E. (2002). Differential expression of calretinin and metabotropic glutamate receptor mGluRlalpha defines subsets of unipolar brush cells in mouse cerebellum. J. Comp. Neurol. 451, 189-199.

Owens, D. F., and Kriegstein, A. R. (2002). Is there more to GABA than synaptic inhibition? Nat. Rev. Neurosci. 3, 715-727.

Pascual, M., Abrasolo, I., MingoranceLe Meur, A., Martinez, A., Del Rio, J. A., Wright, C. V. E., Real, F. X., and Soriano, E. (2007). Cerebellar GABAergic progenitors adopt an external granule celllike phenotype in the absence of Ptfla transcription factor expression. Proc. Natl. Acad. Sci. U.S.A. 104, 5193-5198.

Ponti, G., Crociara, P., Armentano, M., and Bonfanti, L. (2010). Adult neurogenesis without germinal layers: the "atypical" cerebellum of rabbit. Arch. Ital. Biol. 148, 147-158.

Ponti, G., Peretto, P., and Bonfanti, L. (2008). Genesis of neuronal and glial progenitors in the cerebellar cortex of peripuberal and adult rabbits. PLoS ONE 3, e2366. doi:10.1371/journal.pone.0002366

Rakic, P. (1972). Extrinsic cytological determinants of basket and stellate cell dendritic pattern in the cerebellar molecular layer. J. Comp. Neurol. 146, 335-354.

Ramón Y Cajal, S. (1911). Histologie du système nerveux de l'homme et des vertébrés. Paris: Maloine.

Rolando, C., Gribaudo, S., Yoshikawa, K., Leto, K., De Marchis, S., and Rossi, F. (2010). Extracerebellar progenitors grafted to the neurogenic milieu of the postnatal rat cerebellum adapt to the host environment but fail to acquire cerebellar identities. Eur. J. Neurosci. 31, 1340-1351.

Rossi, F., Borsello, T., and Strata, P. (1992). Embryonic Purkinje cells grafted on the surface of the cerebellar cortex integrate in the adult unlesioned cerebellum. Eur. J. Neurosci. 4, 589-593.

Salsano, E., Croci, L., Maderna, E., Lupo, L., Pollo, B., Giordana, M. T., Consalez, G. G., and Finocchiaro, G. (2007). Expression of the neurogenic basic helix-loop-helix transcription factor NEUROG1 identifies a subgroup of medulloblastomas not expressing ATOH1. Neuro-oncology 9, 298-307.

Schilling, K. (2011). "Specification and development of GABAergic interneurons," in Handbook of the Cerebellum and Cerebellar Disorders, eds M. Manto, N. Koibuchi, J. Schmamann, D. Gruol, and F. Rossi (Berlin, NY: Springer)

Schilling, K., Oberdick, J., Rossi, F., and Baader, S. L. (2008). Besides Purkinje cells and granule neurons: an appraisal of the cell biology of the interneurons of the cerebellar cortex. Histochem. Cell Biol. 130, 601-615.

Sekerkovà, G., Ilijic, E., and Mugnaini, E. (2004). Time of origin of unipolar brush cells in the rat cerebellum as observed by prenatal bromodeoxyuridine labeling. Neuroscience 127, 845-858.

Silbereis, J., Cheng, E., Ganat, Y. M., Ment, L. R., and Vaccarino, F. M. (2009). Precursors with glial fibrillary acidic protein promoter activity transiently generate GABA interneurons in the postnatal cerebellum. Stem Cells 27, 1152-1163.

Silbereis, J., Heintz, T., Taylor, M. M., Ganat, Y., Ment, L. R., Bordey, A., and Vaccarino, F. M. (2010). Astroglial cells in the external granular layer are precursors of cerebellar granule neurons in neonates. Mol. Cell. Neurosci. 44, 362-373.

Sillitoe, R. V., and Joyner, A. L. (2007). Morphology, molecular codes, and circuitry produce the threedimensional complexity of the cerebellum. Annu. Rev. Cell Dev. Biol. 23, 549-577.
Simat, M., Parpan, F., and Fritschy, J. M. (2007). Heterogeneity of glycinergic and gabaergic interneurons in the granule cell layer of mouse cerebellum. J. Comp. Neurol. 500, 71-83.

Singec, I., Knoth, R., Ditter, M. Frotscher, M., and Volk, B. (2003). Neurogranin expression by cerebellar neurons in rodents and nonhuman primates. J. Comp. Neurol. 459, 278-289.

Sotelo, C., and Alvarado-Mallart, R. M. (1991). The reconstruction of cerebellar circuits. Trends Neurosci. 14 350-355.

Sudarov, A., Turnbull, R. K., Kim, E. J. Lebel-Potter, M., Guillemot, F., and Joyner, A. L. (2011). Ascll genetics reveals insights into cerebellum local circuit assembly. J. Neurosci. 31, 11055-11069.

Sultan, F., and Bower, J. M. (1998) Quantitative Golgi study of the rat cerebellar molecular layer interneurons using principal components analysis. J. Comp. Neurol. 393, 353-373.

Sun, Q. Q. (2007). The missing piece in the "use it or lose it" puzzle: is inhibition regulated by activity or does it acts on its own accord? Rev. Neurosci. 18, 295-310.

Uussisari, M., and Knöpfel, T. (2011) Functional classification of neurons in the mouse lateral cerebellar nuclei. Cerebellum 10, 637-646.

Wang, V. Y., Rose, M. F., and Zoghbi, H. (2005). Mathl expression redefines the rhombic lip derivatives and reveals novel lineages within the brainstem and cerebellum. Neuron 48, 31-43.

Weisheit, G., Gliem, M., Endl, E., Pfeffer P. L., Busslinger, M., and Schilling, K. (2006). Postnatal development of the murine cerebellar cortex: formation and early dispersal of basket, stellate and Golgi neurons. Eur. J. Neurosci. 24, 466-478.

Williams, I. M, Carletti, B., Leto, K., Magrassi, L., and Rossi, F (2008). Cerebellar granule cells transplanted in vivo can follow physiological and unusual migratory routes to integrate into the recipient cortex. Neurobiol. Dis. 30 139-149.
Wingate, R. J., and Hatten, M. E. (1999). The role of the rhombic lip in avian cerebellum development. Development 126, 4395-4404.

Wingate, R. J. T. (2001). The rhombic lip and early cerebellar development. Curr. Opin. Neurobiol. 11, 82-88.

Wonders, C. P., Taylor, L., Welagen, J., Mbata, I. C., Xiang, J. Z., and Anderson, S. T. (2008). A spatial bias for the origins of interneuron subgroups within the medial ganglionic eminence. Dev. Biol. 314, 127-136.

Yamanaka, H., Yanagawa, Y., and Obata, K. (2004). Development of stellate and basket cells and their apoptosis in mouse cerebellar cortex. Neurosci. Res. 50, 13-22.

Zhang, L., and Goldman, J. E. (1996a). Generation of cerebellar interneurons from dividing progenitors in white matter. Neuron 16, 47-54.

Zhang, L., and Goldman, J. E. (1996b). Developmental fates and migratory pathways of dividing progenitors in the postnatal rat cerebellum. J. Comp. Neurol. 370, 536-550.

Zordan, P., Croci, L., Hawkes, R., and Consalez, G. G. (2008). Comparative analysis of proneural gene expression in the embryonic cerebellum. Dev. Dyn. 237, 1726-1735.

Conflict of Interest Statement: The authors declare that the research was conducted in the absence of any commercial or financial relationships that could be construed as a potential conflict of interest.

Received: 14 November 2011; accepted: 03 February 2012; published online: 20 February 2012.

Citation: Leto K, Rolando $C$ and Rossi $F$ (2012) The genesis of cerebellar GABAergic neurons: fate potential and specification mechanisms. Front. Neuroanat. 6:6. doi: 10.3389/fnana.2012.00006

Copyright (c) 2012 Leto, Rolando and Rossi. This is an open-access article distributed under the terms of the Creative Commons Attribution Non Commercial License, which permits noncommercial use, distribution, and reproduction in other forums, provided the original authors and source are credited. 